\title{
Évaluation par analyse multivariate de la conformation de la mamelle chez la brebis Valle del Belice en relation avec la production laitière
}

\author{
Baldassare Portolano ${ }^{\mathrm{a} *}$, Massimo Todaro ${ }^{\mathrm{a}}$, Raffaella Finocchiaro ${ }^{\mathrm{b}}$, \\ Pietro Giaccone ${ }^{\mathrm{a}}$, Giulio Pagnacco ${ }^{\mathrm{b}}$
}

\author{
a Institut de zootechnie générale, faculté d'agronomie, \\ Université de Palerme, Viale delle Scienze, Parco d'Orleans, 90128 Palermo, Italia \\ ${ }^{b}$ Institut de zootechnie vétérinaire, faculté de médecine vétérinaire, \\ Université de Milan, Via Celoria, 1020133 Milano, Italia
}

(Reçu le 2 juillet 1998 ; accepté le 25 juin 1999)

\begin{abstract}
Evaluation by multivariate analysis of udder conformation in Valle del Belice sheep in relation with milk production. In this study a multivariate statistical analysis method was used; putting in evidence the relationships between udder traits conformation and milk production. 125 Valle del Belice breed ewes reared in two flocks of Santa Margherita Belice (Agrigento) were measured. Data of the udder morphological characteristics on ewes of different parity during January, February, March, August, September and December months were collected. Variables with correlation between original variables and principal component factor, higher than 0.50 were kept into the model. Three principal factors which account for $80 \%$ of the total variability, have been determined; the first factor explained $33 \%$ of the total variability, to this factor are associated all production variables : test day milk yield $(0.88)$, total milk yield $(0.62)$, daily milk yield $(0.63)$; and among the udder morphological variables: udder maximum circumference $(0.89)$, udder circumference $(0.62)$ and udder volume before milking $(0.68)$. The second factor explained $26 \%$ of the total variance; to this factor all the variables linked to the udder conformation were retained: udder depth $(0.76)$, distance between udder base and fetlocks (0.72), udder height (0.69), teat angle (0.53) and udder volume after milking (0.50). $21 \%$ of the total variance were explained from the third factor. To this latter, with a high correlation value, all variables responsible of the teats shape: teat length, teat diameter and udder volume after milking, were linked. Scores were assigned to each variable, to create classes for a linear morphological evaluation. Furthermore, animals were classified with a total score obtained by adding the partial score of the described variables. Total linear score values range from 11 to 20 with mean equal to 15.54 . The linear regression coefficient of the test day milk yield and the udder linear score was equal to $135 \mathrm{~g} / \mathrm{score}$ unit; $\mathrm{R}^{2}$ was equal to 0,55 and the correlation coefficient was equal to 0.74 . Results obtained in this study will be tested in a future project with the aim to evaluate the milking machine ability of the Valle del Belice breed ewe. ( Elsevier / Inra).
\end{abstract}

multivariate analysis / udder conformation / milk production / Valle del Belice sheep

* Correspondance et tirés à part.

Tél.: 0003991485713 ; fax : 0003991481425 ; e-mail : zootgen@unipa.it 
Résumé - Dans ce travail, a été étudiée la possibilité d'utilisation d'une méthode d'analyse statistique multivariée pour mettre en évidence les caractères morphologiques les plus liés à la production laitière afin de les utiliser dans l'évaluation morphologique de la mamelle. Ce travail a été réalisé sur 125 brebis de race Valle del Belice, originaires de deux troupeaux de Santa Margherita Belice (Agrigente). Les données sur les caractéristiques morphologiques de la mamelle, chez des brebis à numéros de lactation différents ont été recueillies, au cours des mois de janvier, février, mars, août, septembre et décembre. Chez tous les animaux, les contrôles décrits ci-dessous ont été réalisés à $100 \pm 6 \mathrm{j}$ de mise bas. L'analyse en composantes principales a retenu trois facteurs qui représentent $80 \%$ de la variabilité totale. Le premier facteur représente à lui seul $33 \%$ de la variabilité totale. Ce facteur pourrait être décrit comme le facteur «forme-fonction de la mamelle » auquel sont associées toutes les variables de production : production laitière au contrôle $(0,88)$, production laitière effective $(0,62)$, production laitière moyenne journalière $(0,63) \mathrm{et}$, parmi les variables de morphologie de la mamelle, la circonférence maximum $(0,89)$, la circonférence au point d'attache $(0,62)$ et le volume avant la traite $(0,68)$. Le deuxième facteur qui représente $26 \%$ de la variance totale correspond à tous les caractères responsables de la conformation de la mamelle : la profondeur $(0,76)$, et la hauteur $(0,69)$ de la mamelle, la hauteur de la citerne du lait $(0,72)$, l'inclinaison des trayons $(0,53)$ et le volume après la traite $(0,50) .21 \%$ de la variance totale sont expliqués par le troisième facteur. Dans ce facteur on trouve, avec des valeurs de corrélations très élevées, les variables de la forme des trayons : la longueur, la largeur et le volume de la mamelle après la traite. Cinq des treize variables morphologiques considérées ont contribué à expliquer significativement les relations entre la forme et la fonction de la mamelle. L'analyse en composantes principales et la régression multiple ont permis de différencier les mesures phénotypiques les plus liées à la production laitière : circonférence maximale, profondeur, volume avant et après la traite de la mamelle et longueur des trayons. (@ Elsevier/Inra).

analyse multivariate / conformation de la mamelle / production laitière / brebis Valle del Belice

\section{INTRODUCTION}

Chez les races ovines laitières le développement et les caractéristiques morphologiques de la mamelle, surtout pour la traite mécanique, sont des paramètres importants qui définissent le débit du lait et donc l'aptitude à la traite mécanique. Ainsi, la pratique de l'égouttage manuel ou repasse est un inconvénient majeur qui trouve son origine dans la conformation particulière de la mamelle $[6,10]$.

Sagi et Morag [11] affirment que chez les brebis traites à la machine, le débit du lait est fonction de la conformation des glandes mammaires et ils mettent en évidence que les mamelles avec les trayons les plus petits (plus courts et plus étroits) entraînent, à même volume de la mamelle [9], des résultats moindres que les mamelles avec les trayons plus grands (plus longs et plus larges).
De nombreux auteurs $[1,7,11]$ proposent un classement en fonction de la conformation de la glande mammaire. Les critères de ce classement sont les suivants : forme de la mamelle, hauteur de la citerne du lait, inclinaison, longueur et largeur des trayons. Gootwine et al. [5] et Pilla et al. [8] définissent comme correctement conformées les mamelles avec des trayons bien formés et implantés en position verticale, et avec une hauteur de citerne très faible. Au contraire, d'autres chercheurs démontrent que les caractéristiques morphologiques de la mamelle favorables à la traite mécanique sont différentes de celles recherchées en traite manuelle [2], soulignant que la production laitière est supérieure lorsque les mamelles sont plus volumineuses même si elles sont moins bien conformées [2, 6,9].

Les relations existant entre les caractéristiques morphologiques de la mamelle, l'aptitude à la traite mécanique et la production laitière sont assez difficiles à éta- 
blir et donnent souvent des résultats contradictoires. Dans ce travail, nous avons étudié la possibilité d'utiliser une méthode d'analyse statistique multivariée pour mettre en évidence les caractères morphologiques les plus liés à la production laitière afin de les utiliser dans l'évaluation morphologique.

\section{MATÉRIEL ET MÉTHODES}

\subsection{Mesures expérimentales}

Ce travail a été réalisé sur 125 brebis de race Valle del Belice, originaires de deux troupeaux de Santa Margherita Belice (Agrigente). Les données sur les caractéristiques morphologiques de la mamelle, chez des brebis à numéros de lactation différents ont été recueillies, au cours des mois de janvier, février, mars, août, septembre et décembre. Chez tous les animaux, les contrôles décrits ci-après ont été réalisés à $100 \pm 6 \mathrm{j}$ de mise bas et sont représentés (figures 1 et 2 ):
- circonférence au point d'attache (cat) ;

- circonférence maximale, mesurée au-dessus du point d'attache des trayons $\left(\mathrm{c}_{\max }\right)$;

- hauteur de la mamelle, mesurée entre le point d'attache et le plan le plus bas du pis (hm);

- largeur de la mamelle, mesurée au dessus de la circonférence au point d'attache (Im) ;

- longueur et largeur du trayon (Int et lgt respectivement) droit ;

- inclinaison du trayon droit, mesurée par l'angle en degrés formé par le trayon avec la verticale $(\alpha d)$;

- hauteur de la citerne du lait (hc) ;

- profondeur de la mamelle, mesurée entre l'attache arrière du pis et le point d'attache abdominal (Prof) ;

- distance entre le plan le plus bas du pis et les jarrets : valeurs positives pour les mamelles avec le niveau le plus bas au dessous des jarrets, alors qu'on obtient des valeurs négatives pour les mamelles avec le niveau le plus bas au-dessus des jarrets (Jar) ;
Figure 1. Mesures des caractéristiques morphologiques effectuées sur la mamelle.

Figure 2. Autres mesures effectuées sur la mamelle.
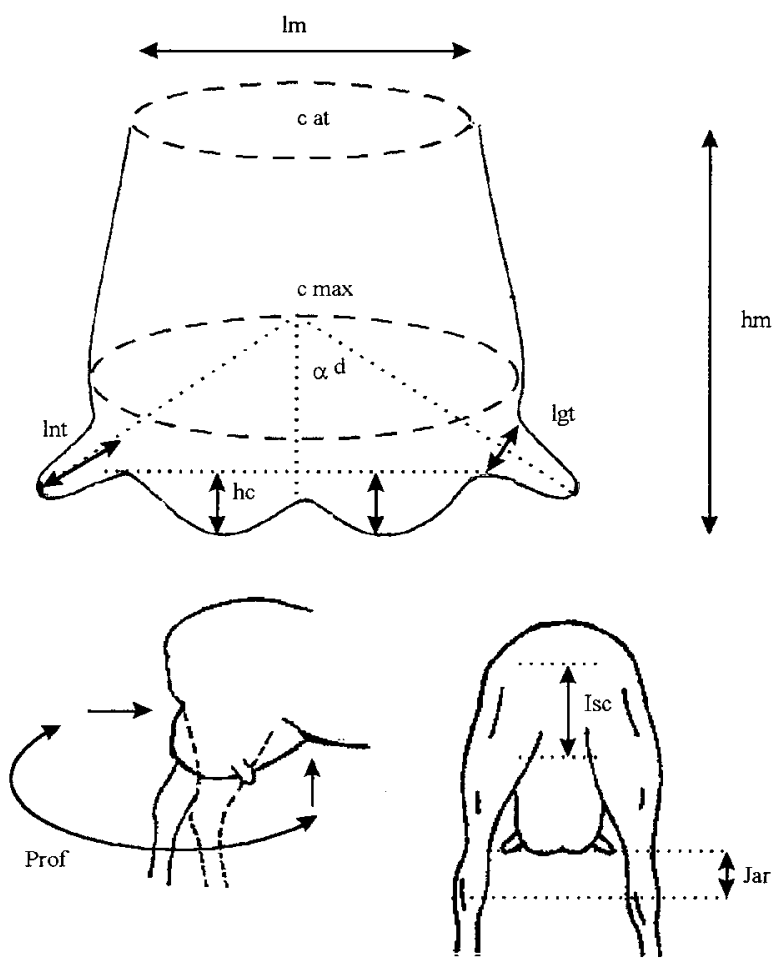
- distance du point d'attache du pis jusqu'à l'ischion (Isc) ;

- volume de la mamelle avant la traite, obtenu en l'immergeant profondément dans un grand bécher en plastique rempli à ras bord d'eau tiède ; en connaissant les poids du bécher avant et après le débordement, il est aisé de calculer le volume de la mamelle $(\mathrm{Vol} \mathrm{I})$;

- volume de la mamelle après la traite, mesuré comme décrit ci-dessus (Vol II) ;

- production laitière du $31^{\mathrm{e}}$ jour après la mise bas jusqu' au tarissement (Prdeff) ; trl) ;

- production laitière à la traite du matin (Prdc-

- production laitière moyenne journalière dans la lactation effective, du $31^{\mathrm{C}}$ jour après la mise bas jusqu'au tarissement (Prdjour).

Les moyennes et les écarts types des mesures obtenues sont présentés dans le tableau l. Pour chaque animal, les données telles que la date, le type de la mise bas et le numéro de lactation ont été relevées.

\subsection{Analyse statistique}

Toutes les données brutes ont été préajustées en utilisant un modèle d'analyse de variance pre- nant en compte les interactions exploitation $x$ mois de mise base ( 12 niveaux) et numéro de lactation (quatre niveaux). Les statistiques descriptives ćlémentaires et les corrélations de Pearson de toutes les variables ont été calculées, puis l'étude statistique a été réalisée en utilisant une analyse en composantes principales.

L'évaluation de la variance commune d'une variable avec l'ensemble des autres variables a été obtenue itérativement par la méthode Iterated Principal Factor Analysis. La matrice de corrélations ainsi obtenue a été diagonalisée par la procédure Varimax. Pour l'individualisation des variables morphologiques les plus liées à la production (ici, la production laitière au contrôle) un modèle de régression multiple avec la méthode d'élimination a posteriori a été utilisé (on retire progressivement de l'équation de prédiction les variables qui ont la plus faible contribution à la prédiction).

En outre il a été supposé une évaluation morphologique linéaire, en utilisant les variables statistiquement significatives lors de l'analyse de régression multiple. Par la suite chacune des variables a été divisée en cinq classes en fonction de leur variabilité. La distribution des scores entre 1 et 5 et le jugement relatif $(1:$ presque suffisant ; 2 : suffisant ; 3 : moyen ; 4 : bon ; 5 : optimal) ont été faits en prenant en compte

Tableau I. Moyenne, écart type, valeur minimale et maximale des caractéristiques morphologiques et production laitière des brebis.

\begin{tabular}{|c|c|c|c|c|}
\hline Variables & Unité de mesure & $\mu \pm \sigma$ & Min & $\operatorname{Max}$ \\
\hline Circonférence au point d'attache (cat) & $\mathrm{cm}$ & $39,2 \pm 5,2$ & 27,6 & 51,5 \\
\hline Circonférence maximale (cmax) & “ & $49,6 \pm 5,3$ & 34,5 & 63,5 \\
\hline Hauteur de la mamelle $(\mathrm{hm})$ & “ & $24,6 \pm 3,9$ & 15,1 & 32,6 \\
\hline Largeur de la mamelle $(l m)$ & “ & $10,9 \pm 1,7$ & 5,5 & 16,0 \\
\hline Longueur de la mamelle $(\ln t)$ & " & $3,5 \pm 0,7$ & 1,8 & 5,5 \\
\hline Largeur des trayons $(\lg t)$ & “ & $2,3 \pm 0,4$ & 1,4 & 3,6 \\
\hline Inclinaison des trayons $(\alpha d)$ & ${ }^{\circ} \alpha$ & $62,3 \pm 16,4$ & 20,0 & 90,0 \\
\hline Hauteur de la citerne du lait (hc) & $\mathrm{cm}$ & $2,7 \pm 1,9$ & 0,0 & 8,8 \\
\hline Profondeur de la mamelle (Prof) & “ & $44,6 \pm 7,1$ & 25,5 & 63,7 \\
\hline Distance bas de trayons - jarrets (Jar) & “ & $2,9 \pm 4,5$ & $-9,5$ & 13,0 \\
\hline Distance attache du pis - ischion (Isc) & “ & $7,3 \pm 1,7$ & 2,6 & 11,4 \\
\hline Volume avant la traite $(\mathrm{Vol} I)$ & $\mathrm{g}$ & $2590 \pm 700$ & 1055 & 4405 \\
\hline Volume aprés la traite ( $\mathrm{Vol} / \mathrm{I})$ & “ & $1654 \pm 400$ & 775 & 3045 \\
\hline Production au contrôle (Prdctrl) & “ & $11,34,2 \pm 365,3$ & 295 & 2160 \\
\hline Production effective (Prdeff) & $\mathrm{kg}$ & $363,5 \pm 131,1$ & 139,7 & 683,6 \\
\hline Production moyenne jouranlière (Prdjour) & $\mathrm{g} / \mathrm{j}$ & $1,7 \pm 0,4$ & 0,97 & 2,78 \\
\hline
\end{tabular}


les signes des coefficients de régression multiple. La somme des scores des différentes variables a permis d'obtenir un score total pour la mamelle de chaque animal.

La régression linéaire et la corrélation entre le score total et la production au contrôle ont ensuite été calculées. L'analyse statistique de l'ensemble de cette étude a été faite à l'aide du Iogiciel SAS 6.12 [12].

\section{RÉSULTATS ETT DISCUSSION}

La moyenne, l'écart type, la valeur minimale et maximale de toutes les variables prises en considération sont présentés dans le tableau $I$.

Le tableau II donne les valeurs des coefficients de corrélation de Pearson entre les variables qui ont été trouvées statistiquement significatives $(p \leq 0,01)$.

Le tableau $I I I$ présente les résultats de l'analyse en composantes principales après rotation de type varimax. Au sein de chaque facteur les variables avec des corrélations supérieures à 0,50 ont été retenues. Le premier facteur représente à lui seul $33 \%$ de la variabilité totale. Ce facteur pourrait être décrit comme le facteur « forme-fonction de la mamelle » auquel sont associées toutes les variables de production : production laitière au contrôle $(0,88)$, production laitière effective $(0,62)$, production laitière moyenne journalière $(0,63)$. Parmi les variables morphologiques, la circonférence de la mamelle au point d'attache $(0,62)$, la circonférence maximale $(0,89)$ et le volume avant la traite $(0,68)$ ont été retenues dans ce premier facteur.

Le deuxième facteur qui représente $26 \%$ de la variance totale correspond a tous les caractères responsables de la conformation de la mamelle : la profondeur $(0,76)$, et la hauteur $(0,69)$ de la mamelle, la hauteur de la citerne du lait $(0,72)$, l'inclinaison des trayons $(0,53)$ et le volume après la traite $(0,50)$.

$21 \%$ de la variance totale sont expliqués par le troisième facteur. Dans ce facteur on trouve, avec des valeurs de corrélations très élevées, les variables de la forme des trayons : la longueur, la largeur et le volume de la mamelle après la traite.

Les coefficients de régression multiple ont été de $44,3 \pm 4,6$ pour la circonférence maximale de la mamelle, $-48,4 \pm 20,7$ pour la longueur du trayon, $12,4 \pm 3,3$ pour la profondeur de la mamelle, $270 \pm 50$ pour le volume avant la traite et $-400 \pm 58$ pour le volume après la traite. Ces cinq variables morphologiques sont en effet celles qui ont été retenues avec la méthode d'élimination a posteriori de la régression multiple et ce sont aussi celles qui, à l'analyse en composantes principales, ont eu les valeurs de communauté les plus élevées : les composantes 1,2 et 3 pour ces variables expliquent une proportion plus élevée de variance totale. (tableau III).

Toutes ces informations ont été utilisées pour procéder à l'évaluation linéaire de la mamelle, comme cela a déjà été proposé par d'autres auteurs [3, 4], en utilisant seulement les variables de référence obtenues par l'analyse factorielle et la régression multiple avec la méthode d'élimination a posteriori. À chacune des variables ont été assignés des scores qui ont constitué les classes d'évaluation morphologique linéaire (tableau IV).

Les variables ainsi classées, on a attribué à tous les animaux un score total obtenu en additionnant le score partiel des variables décrites auparavant. Dans cet essai, les scores totaux qui doivent être compris entre 5 et 25 ont des valeurs entre 11 et 20 et une moyenne de 15,54. Le coefficient de régression linéaire de la production au contrôle et le score total est $135 \mathrm{~g} / \mathrm{unité} \mathrm{de} \mathrm{score;} \mathrm{le}$ $\mathrm{R}^{2}$ de la régression a été de 0,55 alors que la corrélation entre les mêmes variables a été de 0,74. Ce résultat fait supposer, sur cet échantillon de données, la possibilité d'insérer les variables étudiées dans une fiche d'évaluation morphologique linéaire pour le pis, qui pourrait constituer un moyen de sélection efficace pour l'amélioration de la 


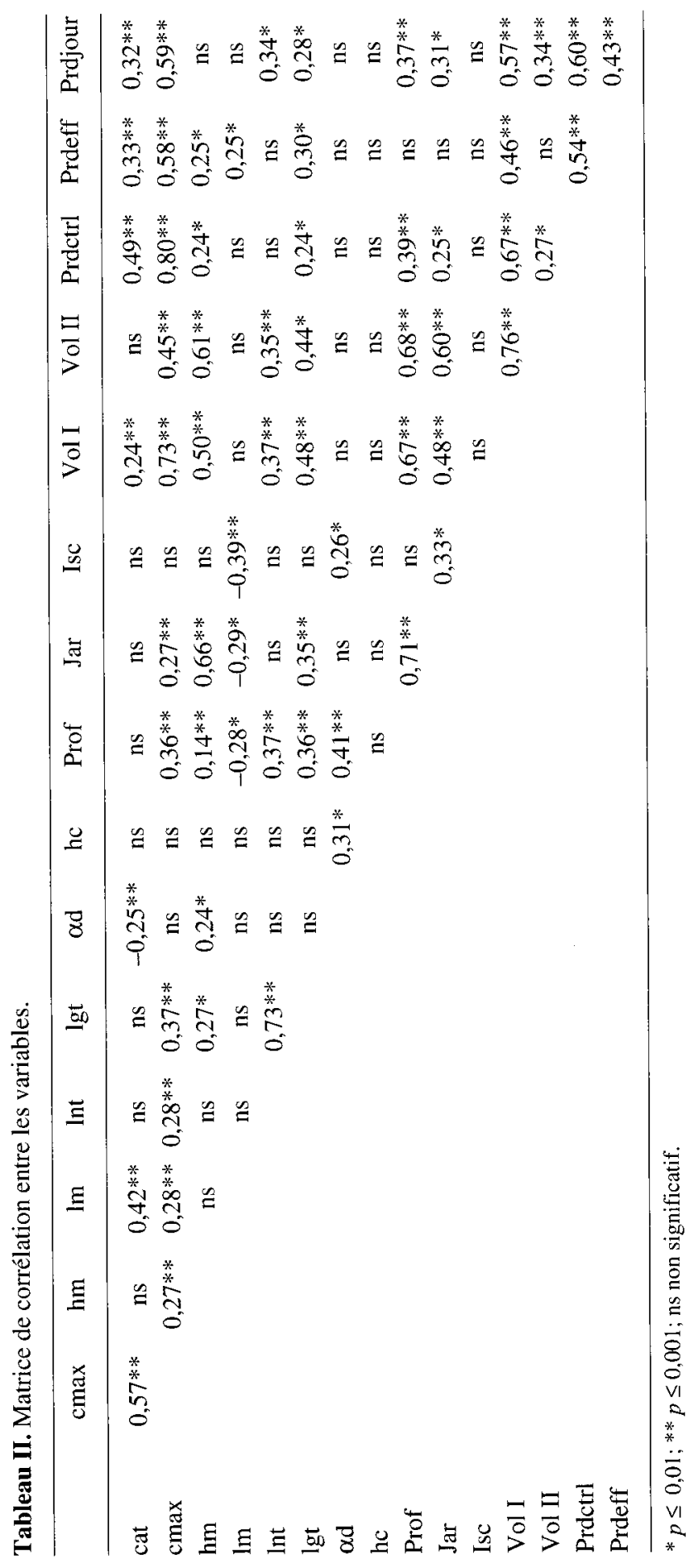


Tableau III. Résultat de l'Analyse en composantes principales.

\begin{tabular}{|c|c|c|c|c|}
\hline \multirow[t]{2}{*}{ Variables } & \multicolumn{3}{|c|}{ Coefficients de corrélations } & \multirow[t]{2}{*}{ Communalités } \\
\hline & Composante & mposante & nposante 3 & \\
\hline cat & $0,62 *$ & $-0,38$ & $-0,04$ & 0,54 \\
\hline $\operatorname{cmax}$ & $0,89^{*}$ & 0,03 & 0,22 & 0,84 \\
\hline$h m$ & 0,24 & $0,69 *$ & 0,28 & 0,60 \\
\hline $\operatorname{lm}$ & 0,36 & $-0,47$ & $-0,11$ & 0,36 \\
\hline $\ln t$ & 0,12 & $-0,02$ & $0,81^{*}$ & 0,68 \\
\hline $\lg t$ & 0,20 & 0,04 & $0,81^{*}$ & 0,70 \\
\hline$\alpha d$ & $-0,08$ & $0,53 *$ & $-0,18$ & 0,32 \\
\hline$h c$ & $-0,04$ & 0,36 & $-0,24$ & 0,19 \\
\hline Prof & 0,31 & $0,76^{*}$ & 0,36 & 0,80 \\
\hline$J a r$ & 0,20 & $0,72^{*}$ & 0,32 & 0,66 \\
\hline$I s c$ & $-0,05$ & 0,42 & $-0,04$ & 0,18 \\
\hline Vol I & $0,68^{*}$ & 0,37 & 0,42 & 0,79 \\
\hline Vol II & 0,35 & $0,50 *$ & $0,50^{*}$ & 0,63 \\
\hline Prdctrl & $0,88^{*}$ & 0,13 & 0,02 & 0,78 \\
\hline Prdcteff & $0,62 *$ & $-0,01$ & 0,21 & 0,44 \\
\hline Prdctjour & $0,63^{*}$ & 0,16 & 0,23 & 0,48 \\
\hline \multicolumn{5}{|l|}{ Variance expliquéc } \\
\hline par chaque facteur & 3,71 & 2,97 & 2,31 & \\
\hline \multicolumn{5}{|c|}{$\%$ de la variance expliquée } \\
\hline par chaque facteur & 33 & 26 & 21 & \\
\hline
\end{tabular}

*Valeurs supérieures à 0,50 .

Tableau IV. Nombre, moyenne et écart type des scores de jugement.

\begin{tabular}{lrrrrrr} 
& \multicolumn{5}{c}{ Score } & \\
\cline { 2 - 6 } Variables & \multicolumn{2}{c}{ Minimal } & Moyen & \multicolumn{2}{c}{ Maximal } & \\
\cline { 2 - 6 } & 1 & 2 & 3 & 4 & 5 & \\
\hline cmax & 11 & 22 & 57 & 32 & 3 & $2,95 \pm 0,94$ \\
Prof & 5 & 28 & 54 & 30 & 8 & $3,06 \pm 0,94$ \\
Int & 4 & 27 & 42 & 44 & 8 & $3,20 \pm 0,96$ \\
Vol I & 13 & 38 & 45 & 24 & 5 & $2,76 \pm 1,01$ \\
Vol II & 4 & 11 & 36 & 59 & 15 & $3,56 \pm 0,93$ \\
\hline
\end{tabular}

Score : 1 presque suffisant ; 2 suffisant ; 3 moyen ; 4 bon ; 5 optimal. 
conformation de la mamelle en relation avec la production laitière, et également en vue de l'application de la traite mécanique chez cette race.

\section{CONCLUSIONS}

Cinq des treize variables morphologiques considérées ont contribué à expliquer significativement les relations entre la forme et la fonction de la mamelle. L'étude, à travers l'utilisation de l'analyse en composantes principales de la structure de covariation des treize mesures morphologiques et des trois mesures fonctionnelles sur les 125 brebis de race Valle del Belice, a fourni une meilleure interprétation des relations existant entre elles.

L'analyse en composantes principales et la régression multiple ont permis de différencier les mesures phénotypiques les plus liées à la production laitière : circonférence maximale, profondeur, volume avant et après la traite de la mamelle et longueur des trayons. Les résultats obtenus dans cet essai, et le modèle d'évaluation linéaire proposé seront vérifiés dans le cadre d'un projet qui aura comme objectif l'évaluation de l'aptitude à la traite mécanique et l'étude systématique des facteurs de variation et des paramètres génétiques des principaux caractères de conformation de la mamelle chez la brebis Valle del Belice.

\section{RÉFÉRENCES}

[1] Casu S., Carta R., Ruda G., Morphologie de la mamelle et aptitude à la traite mécanique de la brebis Sarde, in : III Symposium Internacional de ordeño mecànico de pequeños rumiantes, Valladolid, Majo 1983, pp. 592-603

[2] Casu S., Barillet F., Carta R., Sanna S. Amélioration génćtique de la forme de la mamelle de la brebis Sarde en vue de la traite mécanique: Résultats préliminaires, in : IV Symposium International sur la traite mécanique des petits ruminants, Tel Aviv, 13-19 septembre 1989. pp. $104-133$.

[3] De la Fuente L.F., Femandez G., San Primitivo F. A linear evaluation system for udder traits of dairy ewes, Livest Prod Sci 45 (1996) 842-849.

[4] Fernande\% G., Alvarez P.,San Primitivo F., De la Fuente L.F., Factors affecting variation of udder traits of dairy ewes, J Dairy Sci 78 (1995) $171-178$

[5] Gootwine E., Alef B., Gadesh S.. Udder conformation and its heritabilty in the Assaf (Awassi $\times$ East Friesian) cross of dairy sheep in Israel, Ann Génét Sel Anim 12 (1980) 9-13.

[6] Labussière J., Dotchewski D., Combaud J.F., Caractéristiques morphologiques de la mamelle des brebis Lacaune, Méthodologie pour l'obtention des données, Relations avec l'aptitude à la traite, Ann. Zootech. 12 (1981) 115-136.

[7] Martini M., Verita P., Cecchi F., Valutazione morfologica della mammella nella razza ovina Massese. Risultati preliminari, in : Atti XI Congresso Nazionale Sipaoc, Perugia, 1-4 Juin 1994, Università degli Studi, Perugia, 253-256.

[8] Pilla A.M., Taibi L., Dell'aquila S., Scardella P., Catillo G., Sviluppo e conformazione della mammella di pecore primipare di razza Comisana (C), Delle Langhe (L), Massese (M) e Sarda (S) e di meticce LxC, LxM e LxS, Zoot Nutr Anim 16 (1990) 345-359.

[9] Portolano B., Todaro M., Giaccone P., Morfologia della mammella e correlazioni con la produzione di latte nella pecora Valle del Belice, Zoot.Nutr. Anim 24 (1998) 67-75.

[10] Portolano N. Igiene dell'allevamento ovino e caprino, Edagricole, Bologna 1987.

[11] Sagi R., Morag M. Udder conformation, milk yield and milk fractionation in the dairy ewes, Ann Zootech 23 (1974) 185-192.

[12] Sas (1990) Sas/Stat User's Guide, Volume 1 chap 21, The Factor Procedure SAS Institute INC., Cary, 1990, pp. 773-820. 